\title{
A Rare Presentation of Gaudal Duplication Syndiome in an Adult with No Functional Impairment
}

\author{
Mayuresh M. Deshpande, Pradeep Goil, Anjan Bannerjee \\ From the Department of Plastic Surgery \& Burns, Sawai Man Singh \\ Medical College, Jaipur, Rajasthan, India.
}

\begin{abstract}
:
Caudal duplication syndrome is a rare congenital association between malformations and duplications of the gastrointestinal and genitourinary systems and neural tube defects. Less than 100 cases have been reported in literature worldwide, majority being diagnosed at birth. To date only 2 patients have been reported to have presented in adulthood. We hereby report a case of caudal duplication with complete duplication of urethra, bladder, vagina, uterus, entire hindgut and midgut but with no functional abnormalities. Her only complaint being narrow double vaginal vaults with anticipated difficulty in sexual function. As normal pregnancy has been reported in uterus didelphys, a simple vaginal septal resection and posterior vaginal wall lengthening with incorporation of a 'Z-plasty' was done along with vulvaplasty to exclude the abnormal anal opening from the fourchette.
\end{abstract}

Key words: Neural Tube Defects, Urogenital Abnormalities, Uterus, Urethra, Pregnancy, Humans.

\section{Introduction}

Caudal duplication syndrome (CDS) as described Domiguez et al. in 1993 is a rare association between malformations and duplications of the gastrointestinal and genitourinary systems and the neural tube defects [1]. Although less than 100 cases have been reported in literature worldwide since 1953, a large variation in presentation have been noted. It is a rare congenital malformation with frequency not well known. The pathophysiology is complex and aetiology unknown and majority of them being diagnosed in neonatal or in paediatric age group [1]. One adult female was reported to have diagnosed with CDS while evaluation for her infertility but she too had undergone unknown surgery in childhood for possible anal malformation [2]. We here represent an adult female with hitherto no medical or surgical history who presented to us for the first time at the age of 23 years for genital deformity correction as she was getting married.

\section{Case Report}

A 23 year old female presented to our OPD for correction of deformity of her external genitalia as

Corresponding Author: Dr. Mayuresh M. Deshpande

Email: mayureshdeshpande14@gmail.com

Received: July 6, 2014 | Accepted: August 8, 2014 | Published Online: August 30, 2014

This is an Open Access article distributed under the terms of the Creative Commons Attribution License (creativecommons.org/licenses/by/3.0)

Conflict of interest: None declared | Source of funding: Nil | DOl: http://dx.doi.org/10.17659/01.2014.0081 
she was going to get married. She gave history of double vaginal, urethral and anal openings since birth. She gave history of two separate streams of urine during micturition since birth with no history of urinary incontinence, retention or other urinary disturbances. She gives history of double anal openings since birth, with an anterior abnormal anus opening into the posterior portion of her vulva, whereas the second anal canal opened in the normal anatomical location. There was no history of any bowel habit disturbances.

There was no significant medical or surgical history in her past. Her only complaints at presentation were the presence of two small vaginal openings in place of a single vagina, with expected difficulty in marriage. She also complained of an abnormal, large skin tag lying between the two vaginal openings and the aberrant anal opening.

On examination, patient appeared to be adequately nourished with weight and built appropriate for age. There was no evidence of any skeletal abnormality or deformities of spine. On examination of the genitalia, the labia appeared to be normal. The clitoris was bifid, with two urethral openings placed transversely adjacent to each other just posterior to it. Behind the urethral openings were the two vaginal openings separated by a thick longitudinal septum, which divided the vaginal vaults into two, each admitting a single finger. The abnormal anterior anal opening was visualised in the fourchette with its anal canal directed anteriorly. There was large $2 \times 3 \mathrm{~cm}$ skin tag lying between the vaginal introitus and the aberrant anal opening [Fig.1]. The second anal opening was visualised at the normal anatomical location of the anus. Sphincter tone in both the openings appeared to be normal.

Ultrasound of pelvis showed uterus didelphys, with ipsilateral fallopian tubes opening into respective uterine cavities which continued as duplicated cervix opening into respective vaginal vaults separated by a longitudinal septum. There was duplication of urinary bladder with duplication of urethra but normal appearing single kidneys were visualised on either side with single ureters draining into their respective bladders. Other solid viscera appeared to be normal. Micturating cystourethrogram showed, duplication of urinary bladder with each bladder draining into their respective urethrae [Fig.2]. Barium studies showed a normal foregut, but duplication of the entire colon draining into their respective rectums and anal canals. Small bowel proximal to the ileo-caecal valve could not be assessed [Fig.3]. Magnetic Resonance Imaging (MRI) of the abdomen confirmed the above findings. There appeared to be duplication of distal ileum but the proximal extent could not be assessed.

As requested by the patient, vaginoplasty was done with resection of the vaginal septum and lengthening of the posterior vaginal wall by incorporation of a Z-plasty using the septal mucosal flaps. The sentinel tag was excised. Vulvaplasty was done by raising skin only flaps from the posterior vulva, and interposing them between the introitus and the aberrant anal opening, and thus excluding the anal opening from the fourchette [Fig.4]. Post-operative period was uneventful, the patient recovered well.

\section{Discussion}

Although caudal duplication syndrome, is described as a rare association between malformations and duplications of the gastrointestinal and genitourinary systems and the neural tube defects only 13 patients have been described to have complete genitourinary duplication [3], with majority of patients being diagnosed at or soon after birth. To our knowledge there have been no reported cases of 'asymptomatic' presentation of CDS in adult hood. There have been two case reports with presentation in adulthood but they both have had surgical intervention during their childhood for suspected anal duplication [2]. 


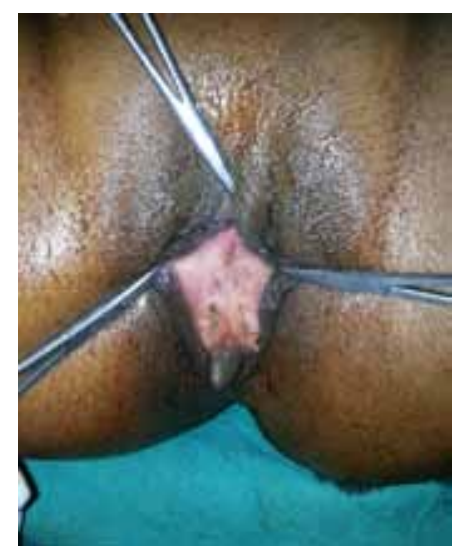

Fig.1: External genitalia of the patient with two transversely placed urethral openings, two vaginal openings and skin tag lying between the vaginal openings and the fourchette. The abnormal anal opening cannot be visualised as it is hidden behind the skin tag.

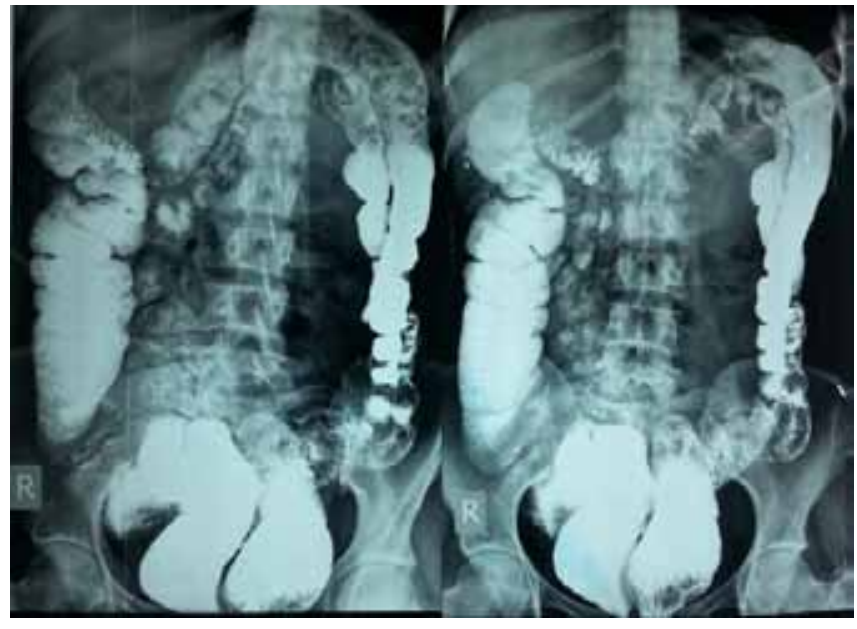

Fig.3: Barium enema showing duplication of anal canal, rectum and entire colon upto the ileo caecal valve.

Greenberg et al. demonstrated that patients can undergo successful vaginal delivery after cloacal malformation repair as infants [4]. Dominguez et al. introduced the term "caudal duplication syndrome" [1], while describing a series of 6 patients who presented with duplication anomalies of various cloacal and caudal neural structures including

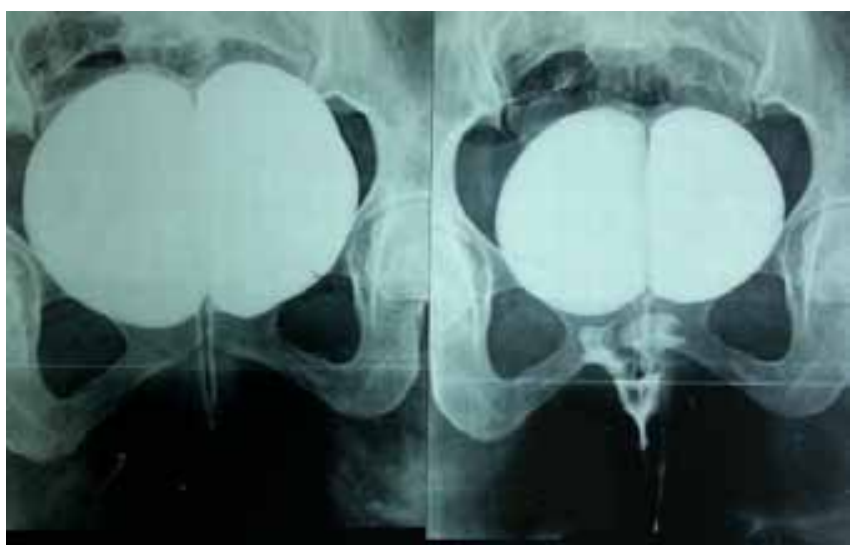

Fig.2: Micturating cystourethrogram showing duplication of bladder and urethrae.

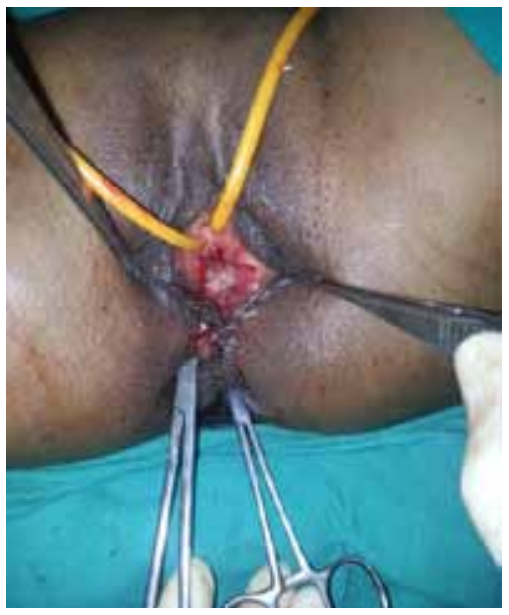

Fig.4: Post-operative photograph showing double urethral openings with foley's catheters in situ, single adequate vaginal opening, skin tag excised and vulvoplasty done exteriorizing the aberrant anterior anal canal opening (indicated by the hemostat on right) and the normal posteriorly opening anal (indicated by the hemostat in midline).

anomalies of varying severity in the genitourinary system, hindgut, and lower spine and cord. In classic cases of CDS, in addition to duplication of the structures derived from the primitive intestine, there are malformations of the spinal column due to damage of the notochord [5]. However, in the 
patient reported here, because there were no neural tube defects, it is expected that the initial anomaly occurred only at the level of the primary intestine, which forms at approximately the fourth week [5].

Although an etiology of caudal duplication is not known, some have espoused a theory of caudal twinning [6]. Embryologically, the primordial hindgut forms as a caudal structure, with the lumen formed by vacuolization, giving rise to the distal ileum, colon, rectum, bladder, and urethra. Abnormal vacuolization of the pluripotent hindgut cell mass during caudal growth might create two parallel hindgut channels, thereby duplicating all hindgut structures distal to the point of separation [7].

A variety of presentations and symptoms have been reported within the spectrum of CDS, uncommonly, this entity is encountered incidentally $[1,8]$. Abnormalities of the external genitalia present with urinary tract obstruction or infection, urinary incontinence, voiding disorders, and anorectal malformations [2]. Uterine abnormalities range from bicornuate uterus to complete uterine and vaginal duplication. Similarly, duplication of the urinary tract can vary in severity from partial to complete duplication of the bladder to lower urinary tract. Anomalies are often associated and usually involve the hindgut. Spinal cord, or spinal deformities have also been reported $[9,10]$. Possible associated hindgut anomalies include duplication of the distal gastrointestinal tract, anal atresia, anal stenosis, and imperforate anus $[2,9,10]$. In our patient, clear duplication of the hindgut and the genitourinary tract, with the absence of skeletal abnormality, was visualised with no functional impairment.

\section{Conclusion}

This is the first case of caudal duplication syndrome to be reported with no functional abnormality and presentation as an adult. In the absence of any functional impairment, only vaginal septal resection and vulvaplasty fulfilled the requirements of the patient.

\section{References}

1. Dominguez $R$, Rott J, Castillo $M$, Pittaluga RR, Corriere JN Jr. Caudal duplication syndrome. Arch Pediatr Adolesc Med 1993;147:10481052.

2. Omar Ragab, Melanie Landay, Jabi Shriki. Complete cloacal duplication imaged before and during pregnancy. Radiology Case 2009;3(11):24-28.

3. Salman $A B$. Cloacal Duplication. Journal of Pediatric Surgery 1996;31(1 1):1587-1588.

4. Greenberg JA, Hendren WH. Vaginal delivery after cloacal malformation repair. Obstet Gynecol 1997;90(4 Pt 2):666-667.

5. Bajpai M, Das K, Gupta AK. Caudal duplication syndrome: more evidence for theory of caudal twinning. Journal of Pediatric Surgery 2004;39(2):223-225.

6. Turan O, Hirfanoğlu IM, Bozdemir P, Baykaner K, Poyraz A, Türkyılmaz $Z$, et al. A rare congenital anomaly: split notochord syndrome with rectal duplication and uterus didelphus. Tip Dergisi / Gazi Medical Journal 2009:Cilt 20:Sayı 3:142145.

7. Kottra JJ, Dodds WJ. Duplication of the large bowel. AJR 1971;113:310-315.

8. Gastol P, Baka-Jakubiak $M$, SkobejkoWlodarska L, Szymkiewicz C. Complete duplication of the bladder, urethra, vagina, and uterus in girls. Urology 2000;55:578-581.

9. Ravitch MM. Hind gut duplication- doubling of colon and genital urinary tract. Ann Surg 1953; 137:588-601.

10. Veeraraghavan KA, Gonzales ET Jr, Gibbons MD. Cloacal duplication: genitourinary and lower intestinal implications. J Urol 1983;129:389 391. 